\title{
Die Einführung von Mitarbeiter/innengesprächen an österreichischen Universitäten
}

\author{
Heidi Möller, Claudia Meister-Scheytt, Gabriela Edlinger, \\ Innsbruck
}

\section{Formen und Wirkungen des Wandels}

Dynamiken des Wandels sind vielfach beschrieben. Der Begriff „Change-Management" hat Hochkonjunktur - so sehr, dass man den Begriff kaum noch hören mag. Die viele Menschen beim Thema des organisationalen Wandels ereilende Abwehr gilt dabei weniger dem Terminus selbst als den mit Wandlungsdynamiken einhergehenden psychischen Prozessen.

Organisatorische Veränderungen können grundsätzlich zwei unterschiedliche Qualitäten aufweisen (vgl. Argyris, Schön 1999, 35; Watzlawick et al. 1992, 29f.). Ein Wandel erster Ordnung zielt ,auf eine inkrementelle Verbesserung, Effektivierung und Anpassung einer Organisation in Anbetracht zu lösender Probleme oder Aufgaben, ohne dabei die dominanten Bezugsrahmen oder Interpretationsschemata zu verändern. Veränderung hat dabei den Charakter von Handlungs- bzw. Fehlerkorrekturen und trägt zur alltäglichen Reproduktion einer Organisation bei. Die Reichweite des Wandels erster Ordnung erstreckt sich allenfalls auf die Ebene der organisatorischen Reproduktionsdynamik" (Becke 2005, 14). Von diesem Veränderungstypus ist der Wandel zweiter Ordnung zu unterscheiden, der den gesamten Bezugsrahmen (oder zumindest hinreichend wichtige Teile) einer Organisation verändert und daher auf einer Metaebene ansetzt und dort Veränderungen hervorbringt. Durch ihn verändert sich ein ganzes System qualitativ. Veränderungen auf der Metaebene schließen prinzipielle Umgestaltungen von bisher allgemein anerkannten sozialen und organisatorischen Vorgehensweisen in Organisationen ein. Bezugsrahmen und Interpretationsschemata werden verändert oder gar ausgetauscht (Staehle 1999, 900).

Die von Watzlawick et al. vorgeschlagene Differenzierung dieser beiden Typen des Wandels scheint jedoch nicht zwingend antagonistisch: Grauzonen zwischen den beiden Modi des Wandels sind vorstellbar. Zudem kann ein Wandel erster Ordnung jederzeit in einen zweiter Ordnung umschlagen (Schülein 1977, 114). Vor allem wenn eine Organisation ohnedies am Rande des Chaos unter extremen Bedingungen und von Instabilität geprägten Beziehungen agieren muss (vgl. Morgan 1997; Briggs, Peat 1990; Weick, Quinn 1999) oder das soziale Netzwerk in hohem Maße interdependent ist, kann eine Anhäufung von vielen Veränderungen erster Ordnung das komplette System kippen und in einen Wandel auf der Metaebene und damit zweiter Ordnung umschlagen. Auch ohne entsprechende Planung können über einen längeren Zeitraum fortdauernde Veränderungen der Alltagspraktiken eine Organisation sozusagen in einer „Revolution 
der kleinen Schritte" verändern (vgl. Hartley 2002, 400; Weick, Quinn 1999, 377). Auch Becke $(2005,14 \mathrm{f}$.) bezieht sich auf das Veränderungspotenzial stetiger kleiner Neuerungen: „Kontinuierlicher Wandel vollzieht sich zwar in kleinen Veränderungsschritten. Wie aus der Kybernetik und der Chaosforschung bekannt ist, können (kumulative) kleine Veränderungen einen qualitativ neuen Zustand herbeiführen.“

Wenn wir uns noch einmal dem Wandel und den notwendigen Transformationen zuwenden, dann weiß ein jedes Mitglied einer Organisation, dass dies Prozesse sind, denen wir uns unterziehen müssen. Wir haben keine Wahl, es sei denn, wir überantworten die Organisation dem Sterben. Wir müssen also innovativ und kreativ sein, und auf der anderen Seite wissen wir als Mitglieder von Organisationen, wie viele dieser Re-engeneeringprozesse scheitern und/oder im Sande verlaufen. In der Literatur spricht man von bis zu 70\% dieser Projekte, die eben scheitern (Gröger 2004). Das Dilemma des Wandels bzw. der Transformation ließe sich in etwa folgendermaßen skizzieren: Wir wissen kognitiv, dass wir uns ständig in einem dynamischen Wandel halten müssen. Wir wissen zudem, wie viele Change-Management-Prozesse schief gehen, und wir kennen unsere vorbewusste Seite, die eher „Nein, nicht schon wieder“ ruft.

\subsection{Die Transformation der Universitäten in Österreich}

Wir wollen nun zur beispielhaften Verdeutlichung der skizzierten Dynamiken auf jenen Veränderungsprozess eingehen, der sich an den österreichischen Universitäten derzeit vollzieht. Die als Auswirkung der 68er Bewegung erkämpfte ,revolutionäre“ Form der Verankerung demokratischer Prinzipien in den Universitäten hatte - so wissen wir heute - nicht die damals intendierten Folgen, sondern vielmehr im Laufe der Jahre eine verstärkte Bürokratisierung und teilweise massive Inflexibilitäten zur Folge. Demokratisierung als „Multiplikation der Entscheidungslast“ (Luhmann 1992) führte dazu, dass mitunter selbst kleinste Entscheidungen komplexe und aufwändige Prozeduren erforderten („Sitzungsuniversität") und dass inneruniversitär klare Entscheidungsstrukturen und -verantwortlichkeiten fehlten. Die zunehmende Größe des Systems, die Aufgabenfülle und die Problemferne des Ministeriums waren immer heftiger artikulierte Gründe für die Forderung nach einer Verlagerung von Entscheidungskompetenzen und -prozessen vom Zentrum (dem Ministerium) zu den dezentralen Einheiten (den Universitäten). Im Anschluss an diese Forderung und vor dem Hintergrund der Forderungen bestimmter Interessengruppen (z.B. des Professorenverbands) nach Abschwächung der „Überdemokratisierung“ wurde auch der Ruf der Öffentlichkeit, der Medien und der Politik nach einer Reform der Universitäten immer lauter. Auch war es nicht zu übersehen, dass die Universität mittlerweile partiell zu einem korrumpierten System geworden war. Für manche Hochschullehrer/in war das Beamtensalaire mittlerweile zu „pocket money“ geworden, die eigentlichen Verdienste machte man in der Dienstzeit an der Universität vorbei, meist ohne genehmigte Nebentätigkeiten und natürlich auf eigene Rechnung. Hier findet zur Zeit ein begrüßenswerter Abschied im Sinne des Qualitätsmanagements und der Interessen der Steuerzahler/innen statt.

Mit der fortschreitenden Reformierung des öffentlichen Sektors - nicht nur in Österreich - und der zunehmenden Einführung von Managementpraktiken, die aus gewinnorientiert arbeitenden Unternehmen stammen, verschwimmen die Grenzen zwischen diesen beiden Bereichen zunehmend (Cornforth 2003). Die Implementierung des UG 2002 war dabei der letzte Schritt in einer vor etwa 30 Jahren eingeleiteten Reformpolitik. Während im UOG 75 das Ministerium alle Autonomie inne hatte, die Universi- 
tät und ihre Untergliederungen als Zwischenebene faktisch über keine Autonomie verfügten, hatten die einzelnen Professor/innen, abgesichert durch ein entsprechendes Beamtendienstrecht, wiederum große Spielräume auf individueller Ebene. Die im UOG 93 eingeleitete und im UG 2002 vollendete Entwicklung verändert dieses Verhältnis grundlegend und gibt der Organisation Universität, vertreten durch die Rektor/in, volle Autonomie, während im Gegenzug das Ministerium gleichsam Teile seiner Entscheidungskompetenzen abgibt sowie die Hochschullehrer/innen in ihrer individuellen Freiheit beschnitten werden. Das Ministerium zieht sich auf eine Position der Rahmensteuerung über die Leistungsvereinbarungen zurück, und die Hochschullehrer unterliegen nun der Dienstgebereigenschaft der Rektor/in. „Es (das UG 2002, d. V.) ist Ausdruck der Fortsetzung der Reformbemühungen der neunziger Jahre, baut auf diesen Gesetzen auf, führt die seinerzeitigen Ideen konsequent fort und wirkt daher viel weniger kompromisshaft als frühere Gesetze“ (Höllinger, Titscher 2004, 11).

Einen Kulturbruch für die Universität stellt auch die zunehmende Bedeutung und Notwendigkeit von Management dar. Führungspositionen wurden bisher maximal für 2 bis 3 Jahre nach einem Modus der Rotation vergeben bzw. besetzt, und Management schien eher etwas für die Welt jenseits des Elfenbeinturms im „Reich der Notwendigkeiten“ zu sein. Nun konstatieren gängige Managementtheorien, dass Management kein Selbstzweck sein kann, sondern als Methode der Koordination von Handlungen zum Zwecke der Zielerreichung dient. Da Zieldimensionen von Universitäten vielfältig sind, müssen Managementkonzepte und -methoden, die zum Einsatz kommen, nicht nur darauf ausgerichtet sein, einer rein ökonomischen Effizienzsteigerung zu dienen, sondern auch zum Ziel haben, den oben genannten essenziellen Herausforderungen zu begegnen, denen sich Universitäten gegenüber sehen. So sind es z.B. im Bereich der strategischen Organisationsgestaltung von Universitäten vor allem die Ideen des „New Public Management" (vgl. Rhodes 1991), auf deren Grundlage der übliche Instanzenzug öffentlicher Verwaltungen durch Prinzipien der Delegation ersetzt wird und Strukturen nach der anzustrebenden Einheit von Aufgaben, Kompetenz und Verantwortlichkeit gebildet werden.

In diesem durch Spannungsfelder und Dilemmata gekennzeichneten Setting kommt dem Management eine bedeutende Rolle zu. Dies vor allem, weil das Management die vormals vom Ministerium vorgenommene bürokratische Detailsteuerung übernehmen muss. Es kann als unstrittig bezeichnet werden, dass das Einräumen größerer Entscheidungsspielräume für monokratische Organe und die Einschränkung der Kontrollfunktionen kollegialer Strukturen zu einer verstärkten Wirksamkeit individueller Faktoren bei der Leitung von Universitäten und Subeinheiten führt. Insbesondere auf der nach dem UG 2002 sehr machtvollen Ebene des Rektorats und auf der Ebene der Fakultätsleitung durch die Dekane spielen daher die Personen, die in diese Funktionen gewählt werden, eine größere Rolle als jemals zuvor.

\subsection{Bausteine der Universitätsreform und die Bedeutung des mittleren Managements}

Kennzeichnend für die Steuerungslogik des neuen Gesetzes sind Instrumente, die bis dato in Universitäten des deutschsprachigen Raums eher unbekannt waren. Dazu gehören unter anderem: die Gewährung eines dreijährigen Globalbudgets auf Basis einer Leistungsvereinbarung mit dem Ministerium; die Einführung einer neuen, aus dem New Public Management entlehnten Governance-Struktur mit machtvollen Rektor/in- 
nen; die Einführung des kaufmännischen Rechnungswesens und Pflicht zur Rechnungslegung, in Verbindung mit ergänzenden Berichtsformen (Wissensbilanzen) und die Gestaltung der Arbeitsbeziehungen auf Basis prinzipiell privatwirtschaftlicher Dienstverhältnisse.

Die Leistungsvereinbarungen mit dem Ministerium werden in Form von Zielvereinbarungen der Rektor/in mit den Dekan/innen weiter gegeben, die dann mit den einzelnen Institutsleiter/innen ihre jeweiligen Zielvereinbarungen treffen. Die Institutsleiter/innen wiederum führen Zielvereinbarungs- und Mitarbeiter/innengespräche mit allen Organisationsmitgliedern der Subsysteme. Derartige Steuerungsmechanismen, die für Mitarbeiter aus der Wirtschaft selbstverständlich sind, bedeuten an der Universität einen Kulturbruch. Zynisch ausgedrückt heißt dies, dass Menschen, die dagesessen sind und über das Leben nachgedacht haben und meinten, sie haben das Recht, dafür vom Staat alimentiert zu werden, nun Mitarbeiter/innengespräche führen und Zielvereinbarungen abschließen müssen, die sie nun zu genau definierten Leistungen (Kongressbesuchen, Veröffentlichungen usw.) verpflichten. Die Zuteilung des Institutsbudgets basiert auf den kollektiven Leistungen der Institutsmitglieder in den verschiedenen Leistungsbereichen. Durch die Einführung von Mitarbeiter/innengesprächen und individuellen Zielvereinbarungen soll ein Commitment für die strategischen Ziele der Universität erreicht werden.

Dass eine angemessene Reaktion auf die Veränderungen keine einfache Aufgabe ist, ergibt sich schon aus den systematischen Organisationsbedingungen von Universitäten. Die Universität als Expertenorganisation besitzt Eigenschaften, die ihre Führung bzw. ihr Management nicht einfach erscheinen lassen. Unklare Technologien, ambigue und komplexe Zielstrukturen, wechselnde Mitgliedschaften (vgl. March, Olsen 1986), ein Personal mit hochgetriebener Spezialisierung (vgl. Pellert 1999), die Wissensbasierung der Organisation (vgl. Nonaka 1994; Nonaka, Takeuchi 1995) und standesmäßig orientierte Hierarchien (Bourdieu 1988) sind nur einige der Stichworte, mit denen die Besonderheiten universitärer Organisationsrealität beschrieben werden. In der Praxis können sich diese Eigenschaften der Organisation „Universität" so auswirken, dass unterschiedliche Stakeholder jeweils unterschiedliche Erwartungen an die Universität herantragen, dass die Identifikation des wissenschaftlichen Personals auf ihre jeweilige scientific community ausgerichtet ist - nicht jedoch auf die ,eigene“ Universität, oder dass Expert/innen sich nicht top-down führen lassen wollen. Das hat für das Management zum Beispiel zur Folge, dass die Wünsche und Vorstellungen der Vertreter/innen unterschiedlicher Disziplinen- und Fachlogiken simultan gemanagt werden müssen.

Eine besondere Bedeutung bei der Handhabung solcher Problemstellungen kommt dem ,mittleren Management“" $\mathrm{zu}$, welches gewissermaßen den „Transmissionsriemen“ darstellt, der zwischen strategischen Herausforderungen und der Alltagspraxis des Wissenschaftsbetriebs ,vermitteln“ muss. Diese Rolle wird in der betriebswirtschaftlichen Fachliteratur mit dem Begriff der „Sandwich-Position“ beschrieben. Damit besteht eine der Hauptaufgaben der Dekan/innen - bzw. des akademischen mittleren Managements - darin, die jeweils von der obersten und der unteren Führungsebene an sie herangetragenen vielschichtigen und widersprüchlichen Erwartungen so zu managen, dass trotzdem möglichst viele Erwartungen zur Zufriedenheit der Beteiligten erfüllt werden können und somit die für den akademischen Betrieb notwendige intrinsische Motivation des Personals nicht gefährdet wird.

Erschwerend kommt zu dieser Aufgabenstellung hinzu, dass bei den Dekan/innen im Gegensatz zur obersten Führungsebene - in der Regel keine Managementerfahrun- 
gen bzw. -qualifikationen vorliegen (müssen); vielmehr erfolgt die Wahl der Dekan/innen oftmals nach dem Senioritätsprinzip. Das bedeutet, dass viele Prozesse „on-the-job“ und mehr oder weniger hemdsärmelig gelernt und ausgeführt werden (müssen), da es sieht man von vereinzelten Beispielen ab (Thom, Tholen 2004) - außerhalb des angelsächsischen Raums im universitären System keine speziellen Personalentwicklungsprogramme für Aufstiegskandidat/innen als Vorbereitung für ihre künftige Position gibt. Den Leiter/innen von Organisationseinheiten werden durch das Universitätsgesetz 2002 also entweder direkt oder indirekt aus dem Geist des Gesetzes ableitbare neue Aufgaben zugeordnet, für deren Übernahme diese Personengruppe in der Regel nicht systematisch vorbereitet, nicht geschult bzw. trainiert wurde. Auch die Erfahrung als mögliche Quelle von Orientierungswissen stellt angesichts der völlig neuen gesetzlichen wie auch organisatorischen Rahmenbedingungen keine hinreichende Basis gelingenden Führungshandelns dar. Zudem konkurriert diese Personengruppe universitätsintern nun um knappe Ressourcen. Damit wird ein Wettbewerb innerhalb der Universität induziert, der die ehemals gültige kollegiale Solidarität massiv unterminiert.

\section{Das Mitarbeiter/innengespräch und seine kommunikativen Besonderheiten}

Sowohl diese beschriebenen Veränderungen hinsichtlich formeller und informeller Strukturen als auch die oben angeführten Besonderheiten der Organisation Universität bilden die Rahmenbedingungen, innerhalb derer an österreichischen Universitäten Mitarbeiter/innengespräche geführt werden. Die Einführung von Mitarbeiter/innengesprächen ist seitens des Rektorats mit bestimmten Zielsetzungen und zahlreichen Erwartungen z.B. hinsichtlich gesteigerter Arbeitszufriedenheit, optimierter Personalentwicklung und höherer Arbeitseffizienz verbunden. Dass diese positiven Effekte sich allerdings nicht wie von selbst allein durch die Implementierung von Mitarbeiter/innengesprächen einstellen, liegt aus Sicht der Systemtheorie nach Luhmann (2002) auf der Hand, weil es sich bei einer Universität um ein nicht-triviales System handelt, dessen Eigenzustand und -dynamik weder völlig analysierbar noch vorhersehbar ist und dessen Steuerbarkeit damit begrenzt bleibt.

Interventionen durch das Management, wie die Einführung von Mitarbeiter/innengesprächen, stellen für das System Universität eine Irritation dar, die als Fremdkörper zunächst - wie eingangs beschrieben - wahrscheinlicher mit Skepsis beäugt als freudvoll akzeptiert und integriert wird. Auf diese Gefahr mit einer PR-Offensive für die Methode des Mitarbeiter/innengesprächs zu reagieren und die zahlreichen Möglichkeiten des Instruments zu bewerben, liegt nahe, ist aus systemischer Sicht aber kaum ausreichend, denn die weitgehend autonome Expert/innenorganisation Universität verarbeitet Managementeingriffe nach ihren eigenen „Gesetzmäßigkeiten“: Dazu gehört insbesondere auch eine Kluft zwischen wissenschaftlichem und administrativem Personal einschließlich dem Universitätsmanagement sowie eine gewisse Skepsis gegenüber neuen universitären Management-Strukturen (Grossmann, Pellert, Gotwald 2000).

Diese Bedingungen erschweren zunächst die erfolgreiche Etablierung von Mitarbeiter/innengesprächen an Universitäten. Dennoch bleibt dieser Prozess von Beginn an keinesfalls wirkungslos. Bereits vor ihrer Umsetzung führt das Wissen über ihre verpflichtende Einführung in Form ,indirekter Kontextsteuerung“ zu Reaktionen, die sowohl zu Veränderungen auf organisationaler als auch auf der Ebene individuellen orga- 
nisationalen Verhaltens führen. Die Reaktionen können von aktiven Gegenstrategien über die Degradierung des Mitarbeitergesprächs zum Formalakt bis hin zum Bemühen um einen sinnvollen Einsatz dieses Führungsinstruments seitens der Führungskräfte reichen. Von Seiten der betroffenen Mitarbeiter/innen muss insbesondere mit einer Zunahme strategischen Agierens im Sinne von Impression Management gerechnet werden. Die Intensität dieser Bemühungen bewegt sich je nach Positionierung des Instruments in der Organisation (als Personalentwicklungs- und/oder als Leistungsevaluierungs-Instrument) und abhängig vom beruflichen Status der Betroffenen von äußerst gering bei Mitarbeiter/innen mit Beamt/innen-Status bis extrem hoch bei jenen, die als Projektmitarbeiter/innen oder im Rahmen zeitlich befristeter Verträge an der Universität tätig sind.

\subsection{Der Einfluss vordiskursiver Parameter}

Noch bevor die ersten Mitarbeiter/innengespräche tatsächlich geführt werden, zeigt die Einführung dieses Führungsinstruments also bereits Wirkungen. Die geänderten Erwartungen, Kommunikationsprozesse und Verhaltensweisen wiederum spielen für das Gespräch selbst eine wesentliche Rolle. Schließlich werden von beiden Beteiligten sämtliche Vorerfahrungen aus ihrer gemeinsamen Kommunikation, aber auch hinsichtlich der gelebten Organisations-, Abteilungs- bzw. Team-Kultur mit in die Gesprächssituation „Mitarbeiter/innengespräch“ gebracht (Looss 1991, 78f.). Das soziale Selbst („Me“) als Teil der Persönlichkeit bezieht sowohl die Erwartungen seiner Interaktionspartner als auch deren erwartete Verhaltensweisen in die Ausgestaltung seines eigenen Handelns mit ein (Mead 1994), weshalb die Möglichkeit eines offenen Dialogs zwischen Vorgesetzten und Mitarbeiter/innen im Mitarbeiter/innengespräch letztlich eine weitreichendere Frage ist als die des bloßen Verstehens im Gespräch: Was außerhalb des Mitarbeitergesprächs nicht möglich ist, passiert auch während des Mitarbeitergesprächs in der Regel nicht.

Daher setzen Trainings für Vorgesetzte, die Mitarbeiter/innengespräche führen sollen, im Idealfall beim Erkennen der Sinnhaftigkeit und der Bedeutung einer gegenseitigen Rückmeldung, beim Formulieren und Empfangen von kritischen Feedbacks und beim Erlernen eines wertschätzenden Gesprächsverhaltens und aktiven Zuhörens an. Von solchen Fortbildungen ist - sofern sie auf qualitativ hohem Niveau angeboten werden - nicht nur ein positiver Effekt auf die Abhaltung von Mitarbeiter/innengesprächen zu erwarten. Professionelle Coachings zielen auf eine dauerhafte Erweiterung von Deutungs- und Handlungsmustern (Schreyögg 1996) und erreichen damit eine bedeutende Steigerung von Sozialkompetenzen. So können insgesamt positive Auswirkungen auf das Arbeits- und Gesprächsklima sowie eine Entwicklung zum „reflective practitioner" (Schön 1983) erreicht werden.

Doch auch wenn es der Führungskraft gelingt, ein positives Grundklima zu schaffen, ist das hohe Ziel eines erfolgreichen Mitarbeiter/innengesprächs noch immer nicht erreicht. Denn die Vorbereitung der Leiter/innen auf das Mitarbeiter/innengespräch reicht insofern nicht aus, da nach Habermas (1981) eine verständigungsorientierte Einstellung aller an einer Kommunikation beteiligten Akteure die Grundlage für kommunikatives Handeln bildet. Erst das Erfassen der Absichten oder Intentionen des anderen ermöglicht, dass das Besprochene im zukünftigen Handeln berücksichtigt werden kann. Genau diese wichtige Funktion kann das Mitarbeiter/innengespräch aber oft nicht erfüllen, denn ,als Mechanismus der Handlungskoordinierung“ kann Verständigung gemäß der Theorie des kommunikativen Handelns nur funktionieren, ,wenn ein Einver- 
ständnis der Beteiligten aufgrund freier und rational motivierter Zustimmung zustande kommt. In dem Moment, in dem die Zustimmung eines Beteiligten von außen auferlegt ist, durch Sanktionsmöglichkeiten erzwungen, durch Manipulation der Handlungssituation oder durch die strategische Beeinflussung von Entscheidungen erreicht wird, verliert Verständigung die Kraft, Handlungen aneinander anzuschließen“ (Mark-Ungericht, Weiskopf 1993, 93).

Dieser Problematik kann theoretisch entgegengewirkt werden, indem Mitarbeiter/innengespräche nicht verpflichtend, sondern auf freiwilliger Basis implementiert werden. Im ehemals weitgehend Management-fernen universitären Feld führte eine derartige Regelung allerdings eher zum Vergessen des Instruments als zu seinem erfolgreichen Einsatz. Empfehlenswert ist vielmehr, die verpflichtende Einführung mit der Möglichkeit, das Konzept innerhalb verschiedener universitärer Bereiche (zumindest auf Fakultäts- eventuell auch auf Institutsebene) anzupassen, zu verbinden, wobei für die Adaptierung professionelle Unterstützung angeboten werden sollte. Noch besser wäre eine allgemeine Coaching-Möglichkeit für Führungskräfte, denn die Anforderungen an wissenschaftliche Leiter, die im universitären Kontext ihre Position häufig immer noch ausschließlich aufgrund ihrer wissenschaftlichen Leistungen - ungeachtet ihrer Sozialkompetenz oder ihrer Management-Fähigkeiten - innehaben, sind beachtlich, und das Ausmaß ihrer Erfüllung stellt einen ganz wesentlichen Faktor für die erfolgreiche Umsetzung von implementierten Management-Techniken wie dem Mitarbeiter/innengespräch dar.

\subsection{Die Durchführung von Mitarbeiter/innengesprächen: Eine kommunikative Herausforderung}

Die Verantwortung für das Gelingen des Dialogs liegt also in punkto Vorbereitung und Durchführung von Mitarbeiter/innengesprächen relativ einseitig bei den Vorgesetzten. Aber auch „die andere Seite“ geht nicht unbelastet in das Gespräch, welches zentral durch ein hierarchisches Gefälle zwischen den beiden Beteiligten gekennzeichnet ist. Diese „,bestehenden Herrschaftsverhältnisse“ sind nach Habermas (1981) die Grundursache für Störungen des zwischenmenschlichen Dialogs, was sich in strategischem (anstelle von kommunikativem) Handeln niederschlägt. Damit steht wieder u.a. die Gefahr des oben bereits erwähnten Impression Managements im Raum.

Der Tatsache, dass die beteiligten Akteure eventuell „nicht nur verstehen, sondern auch Wirkungen erzielen“ wollen, kann laut Habermas nur entgegengewirkt werden, indem ihre Ursache benannt wird, damit „die unterdrückten Bedürfnisse und Interessen der Beherrschten zur Sprache und damit den Parteien zu Bewusstsein" gebracht werden (Habermas, zit.n. Preglau 1997, 246). Dadurch soll an intersubjektiver Verständigung orientiertes kommunikativen Handelns möglich werden. Die höchste Form der Kommunikation bei Habermas - ein Diskurs (als eine Form der Kommunikation, in der es darum geht, strittige Geltungsansprüche zu klären) - kann hingegen nur in einer idealen, herrschaftsfreien Sprechsituation erfolgen, die ausschließlich im subjektiven Empfinden der Akteure entstehen kann. Das Erreichen eines solch positiven, offenen Gesprächsklimas ist wohl die größte Herausforderung in Mitarbeiter/innengesprächen. 


\section{Erfahrungen einer Institutsleiterin und Dekanin mit der Einführung der Mitarbeiter/innengespräche}

Die Hochschullehrer/in von heute ist eine „eierlegende Wollmilchsau“: Gleichzeitig soll er/sie Spitzenleistung in Forschung, Lehre, in der Mitarbeiter/innenführung und natürlich auch in der universitären Selbstverwaltung bringen. Auf der subjektiven Erlebnisebene bedeutet dies ein ständiges Managen von Insuffizienz. Die Arbeitsprofile haben sich komplett verändert. Professor/innen sind jetzt Projektmanager/innen, die für ihre interne und externe Reputation sorgen und die „richtigen“ Allianzen bilden müssen. Daneben sind sie mobil, kooperieren international und gehen regelmäßig zu den für sie relevanten Kongressen. Die Leistungslatte, die früher die ,akademischen Superstars" vorlegten, gilt jetzt für alle gleichermaßen.

Diese Veränderungen finden vor dem Hintergrund der Abschaffung des Beamtenstatus für Hochschullehrer/innen statt: Erfolgreich sein unter Unsicherheit, so das Motto, denn auch Professor/innen haben unter dem neuen Gesetz zunächst nur Vier-, Fünfoder Sechsjahresverträge. $\mathrm{Ob}$ man einen Anschlussvertrag erhält, der dann wieder befristet oder aber auch unbefristet sein kann, hängt ab vom jeweiligen Rektorat, der Performance des Kandidaten und davon, ob die Hochschulleitung in dessen Bereich noch finanzielle Mittel investieren will. Kein komfortables Szenario also für eher risikoaverse Persönlichkeitsstrukturen, wie sie bei Hochschullehrer/innen häufig zu finden sind.

Vor diesem Hintergrund regt sich Widerstand gegen die verschiedenartigen Techniken indirekter Steuerung, die an der Universität wirksam sind und mittels derer der Versuch unternommen wird, die erweiterten Spielräume entgrenzter Arbeits- und Betriebsvorgänge in neuer Form im Griff zu behalten. Im Turbokapitalismus managen sich die Mitarbeiter/innen vor allem selbst. Dies geschieht besonders durch Zugriff auf die emotionale Welt der Arbeitenden (vgl. Hochschild 1990). Gefühle wie Angst (vor dem Wandel, vor dem Verlust des Arbeitsplatzes, dem Tempo nicht zu genügen, der Konkurrenz nicht standzuhalten), schlechtes Gewissen und Hochgefühl, werden Schmidt (2000) folgend systematisch und manipulativ eingesetzt. Aber sind das Argumente gegen Mitarbeiter/innengespräche?

\subsection{Die Gefahr der strukturellen Oszillation}

Ein wichtiges organisationales Moment innerhalb von Mitarbeiter/innengesprächen mit dem gesamten wissenschaftlichen Personal ist die Gefahr einer strukturellen Oszillation im Wandlungsprozess (Fritz 1999): Auf der einen Seite lässt sich ein Veränderungsbedürfnis von Organisationen ausmachen, und es gibt immer wieder die Notwendigkeit zu Reformen, die dann Veränderungsbemühungen in Gang setzt, durch die wiederum Diskontinuitäten entstehen. Gleichzeitig wird im Wandel das Bedürfnis nach Kontinuität freigesetzt und gerät in die affektive Welt der Organisationsmitglieder. Dieser Prozess geht dann einher mit der Vermeidung von Veränderung. Man versucht sich $\mathrm{zu}$ widersetzen, versucht Notwendigkeiten zu ignorieren. Organisationsmitglieder versuchen alles, was an Veränderungsdynamik da ist, zu umgehen, bis dann erneut ein Veränderungsbedürfnis entsteht - eine spiralförmige Bewegung. Plötzlich ist da ein neuer Managementansatz, und die Organisationsmitglieder springen auf die Veränderung an.

Die Pendelbewegung zwischen Wandel und Kontinuität ist zunächst einmal nahezu naturwüchsig. Problematisch ist jedoch, wenn sich die Aktivitäten der Veränderung und 
des Bewahrens gegenseitig nivellieren. Dies führt zum objektiven Entwicklungsstillstand einer Organisation, bei gleichzeitig immensem Kräfteverschleiß. Der Eindruck, ständig auf der Stelle zu treten, geht einher mit einem hohen emotionalen Aufwand. Beides, die Stagnation wie auch die Veränderung, erzeugt Widerstände. Diese gilt es in Mitarbeiter/innengesprächen herauszuarbeiten, zu verstehen und nach Möglichkeit aufzulösen.

Diese organisationalen Prozesse können in einem Mitarbeiter/innengespräch einer gemeinsamen Reflexion unterzogen werden. Damit eine Organisation aber nicht beginnt zu oszillieren und bei jeder Innovation wieder mit ganz viel Unlust in die Gegenrichtung gezogen wird, braucht es ein übergeordnetes Ordnungsprinzip, auf das man sich einigen kann. Sonst besteht die Gefahr, bei Unlustgefühlen immer den Weg des geringsten Widerstandes zu gehen, der eben keine Innovation ermöglicht. Sowohl die Mitarbeiter/innen als auch die Führungskräfte bekommen während des Change Managements oft „,kalte Füße“: „Oje! Das ist jetzt zu viel, das schaffen wir nicht, da gehen wir gleich wieder zurück!" Sie machen viel Wirbel, aber die Energie wird durch diese Unentschiedenheit nivelliert, und die Organisationsmitglieder sind frustriert und unlustig. Beide Spannungs- und Auflösungssysteme wollen siegen, und jedes System sucht nach Gleichgewicht.

Um aus der strukturellen Oszillation zu entkommen, darf sich weder die Führungskraft noch die Mitarbeiter/in auf eine der beiden Seiten zwischen hier Veränderung und da Stagnation schlagen. Wenn die Organisation hin und her oszilliert, kann es keine sinnvolle Entwicklungsrichtung geben. Es braucht einen dritten Bezugspunkt bei gleichzeitiger und gleichmäßiger Entfernung vom Entweder und vom Oder. Der dritte Bezugspunkt können die Werte der Organisation sein, ihr Leitbild (unter Einbezug der organisationalen Paradoxien; vgl. Giesecke 1996), aber auch Volitionen des Managements. Nur so kann es gelingen, die konstruktive Spannung zu halten und handlungsfähig unter Nichtauflösung dieser Spannung zu bleiben. Wir können intern oder durch externe Beratung ein Bewusstsein und Anerkenntnis dieser Spannung herstellen und an einem übergeordneten Ordnungssystem arbeiten, mag es eine Entscheidung oder eine Vision sein, an die zu mahnen ist. Sonst verbraucht sich der Veränderungsprozess selbst, frisst sich von /innen auf.

\subsection{Die Gefahr der Heuchelei in den Mitarbeiter/innengesprächen}

Eine der Möglichkeiten, auf die Herausforderungen der erhöhten Leistungsanforderungen an der Universität zu reagieren, ist der Mechanismus des „Heuchelei-Managements“. Es wird versucht, die verschärften Anforderungen der Systemumwelt durch Heuchelei, einem „So-Tun-Als-Ob“ zu beantworten. Da das Ministerium mit seiner Gesetzesreform die Hoffnung verknüpfte, dass Österreichs Universitäten bald Weltklasse sein würden, richtete man seitens des Ministeriums eine entsprechende Homepage ein (http://www.weltklasse-uni.at). Folglich wird an den Universitäten nun darüber gesprochen, dass man eine Weltklasseuniversität sei. Das offizielle Wording lautet: „Wir sind Weltspitze!“"

Dieses Impression Management ist dabei aber kein einfaches, vielleicht lächerliches Wortspiel, sondern zeitigt massive Auswirkungen auf die Organisationsrealität und vor allem auf die Emotionen der Organisationsmitglieder, die ja vor allem als organisationsrelated anzusehen sind. So wird z.B. universitären Stipendiaten bei der Verleihung der Dekrete verkündet (es handelt sich um Förderbeträge für die Dissertation von $€$ 605,- 
pro Monat für eine maximale Laufzeit von 24 Monaten), sie müssten in einem Jahr fünf Veröffentlichungen bringen, wenn sie nach 12 Monaten eine Verlängerung des Stipendiums um weitere 12 Monate anstreben. Weinende Doktorand/innen, die schon kündigen wollten, galt es am Tag nach der Feierlichkeit zu trösten. Wenn eine tief empfundene Insuffizienz abgewehrt werden muss und mit narzisstischen Größenvorstellungen kompensiert wird, entfaltet sich eine destruktive Dynamik. Dieser gilt es auf der Ebene des akademischen mittleren Managements (der Dekan/innen) sinnvoll und konstruktiv zu begegnen. Insuffizienzgefühle der Leitung können vielleicht mühsam durch narzisstische Phantasien über sich selbst und die Organisation dürftig kompensiert werden.

Dieser Abwehrmechanismus der Organisation führt aber dazu, dass die verleugneten Gefühle an anderer Stelle empfunden werden. Im Sinne einer projektiven Identifikation ${ }^{1}$ bieten sich zumeist weibliche Bedienstete und sensible Mitarbeiter gerade in recht narzisstisch anmutenden Unternehmen als Container negativer abgespaltener Affekte an. Narzissmus im Unternehmen wird hier als Kompensationsmechanismus begriffen. Volkan \& Ast (1994) sprechen davon, wie das hungrige, entwertete, abgespaltene Selbst mit dem Gewand der Großartigkeit daher kommt. Die zunehmende Anspannung, der Arbeitsdruck in Zeiten knapper werdender materieller Ressourcen tun ihr Übriges dazu, dass Bedürftigkeit, Unsicherheit und Angst in solchen Organisationen von Führungskräften nicht wahrgenommen werden. Sensibilität und Sensitivität von empfänglichen Mitarbeiter/innen in solchen Unternehmen bilden nun eine Art Container dieser abgewehrten Empfindungen. Das Abgewehrte, Unbewusste der Organisation, die wirtschaftliche Bedrohung, der gesteigerte Arbeitsdruck u.v.m. finden sich in deren Gefühlsleben wieder. Qua projektiver Identifikation repräsentiert sich das Abgewehrte der Organisation im Seelenleben der Mitarbeiter/innen als eigene subjektive Insuffizienz, die sich in stetigen Selbstzweifeln und Kompetenzzweifeln äußert.

Um diesen destruktiven Auswüchsen zu begegnen, wählte $i^{2}{ }^{2}$ als Dekanin nach den Erfahrungen des Jahres 2005 für das Jahr 2006 nun die Strategie hin zum ,good enough" in der gesamten Fakultät, aber auch in jedem einzelnen Mitarbeiter/innengespräch. Das Motto war: „Wir machen 2006 überhaupt nichts Neues, wir haben 2005 so viel Veränderung vollzogen, dass jetzt die Zeit der Konsolidierung ansteht. Wir werden bei den Zielvereinbarungen lediglich die Kapazität bei $60 \%$ unserer Arbeitskraft festlegen, um den Rest frei verfügbar für eine Reaktion auf die Anforderungen aus der Systemumwelt zu haben." Die Anspannung der Mitarbeiter/innen war wie weggeblasen, und das Zutrauen und die Arbeitsfähigkeit wuchsen deutlich an. Das Motto des ,good enough" (vgl. Winnicott 1991) erwies sich als gangbarer Ausweg aus dem Heucheleimanagement. Es zeigte sich als förderliche Haltung, so förderlich wie das Wording von der Weltspitze hinderlich war und die Menschen unglücklich und die Leistung schlechter werden ließ.

Es war mir auch möglich, mich dem Wunsch des Rektorats zu widersetzen, das Budget kennzifferngesteuert auf jede Mitarbeiter/in herunterzubrechen. Ich halte es für kontraproduktiv, in einer Fakultät, die sich gerade auf den Weg gemacht hat, vom wis-

1 Was ist eine projektive Identifikation? Zur Illustration ein drastisches Beispiel: Zwei Reisende sitzen sich im Zug gegenüber: der eine döst, dem anderen wird übel und er speit dem Schläfrigen auf den Schoss. Als dieser erwacht und die Bescherung sieht, fragt ihn der andere voller Besorgtheit: „Ist Ihnen wieder gut?“ (frei nach Andreatta)

2 Für den folgenden Teil der Reflexion wird von der Dekanin als Agentin des Wandels die „Ich“-Form gewählt. 
senschaftlichen Einzelkämpfer/innentum hin zu mehr team- und kooperationsbezogener Forschung zu kommen, an dieser Stelle des mühsamen Entwicklungsprozesses, den Konkurrenzgedanken zu implementieren.

Nach zwei Jahren der Erfahrung mit Mitarbeiter/innengesprächen kann ich sagen, dass ich sie nicht missen möchte. Obwohl ich in der Rolle als Institutsleiterin eine Menge Personalentwicklungsinstrumente einsetzte: Teamentwicklung, Führungsfeedback, Teamrollenanalyse, ganztägige strategische Sitzungen zu Fragen der Lehre und der Forschung, zweitägige Fakultätsklausuren u.v.m., ist die Begegnung in einem geschützten Rahmen des Vier-Augen-Gesprächs von einer ganz anderen Qualität gekennzeichnet als reflexive gruppenbezogene Settings. In der vertrauensvollen dyadischen Situation gelang es uns (mit einer Ausnahme), ein vertieftes Verständnis füreinander zu entwickeln:

- Fragen der unterschiedlichen Arbeitsstile konnten akkordiert werden. So beschwerte sich eine Mitarbeiterin massiv darüber, dass ich ständig mit neuen Ideen in ihr Büro rausche, sie überschütte mit meinen Impulsen und schließlich konfus zurücklasse. Ich konnte mich auf ihre Notwendigkeit, step by step zu arbeiten und eben kein Multitasking zu können und zu wollen, angemessener einstellen.

- Selbst- und Fremdbilder konnten in einen Dialog überführt werden und ggf. korrigiert werden.

- Voreilige Urteile auf beiden Seiten konnten revidiert werden.

- Auch aggressive Impulse konnten Platz greifen und in produktive Arbeitsenergie umgewandelt werden, sachgerechte Kritik ebenso wie Lob gegenseitig benannt werden.

- Offene Fragen aneinander konnten gründlich und ohne Zeitdruck beantwortet werden.

- Heikle gesundheitliche Probleme und deren Behandlungsnotwendigkeiten konnten im Sinne eines Gesundheitscoachings angesprochen werden.

- Die Entwicklungspotenziale wurden beschrieben und in konkrete Karriereplanungsschritte heruntergebrochen.

- Problematische Arbeitsbeziehungen konnten entweder entschärft oder der Konflikthintergrund sogar aufgelöst werden.

- Neue interne Steuerungsprozesse, die helfen sollen, die Prozesse zu optimieren, wurden gemeinsam ,erfunden“. Bessere, konfliktentschärfende Controllingmöglichkeiten konnten gefunden werden.

- Gegenseitige ungünstige Rolleneinsteuerungen konnten aufgelöst werden.

- Jede Mitarbeiter/in verließ den Besprechungsraum mit klaren Zielen für das nächste Jahr, und die Institutsatmosphäre - auch durch die geklärten Erwartungen aneinander - besserte sich enorm.

Die meisten meiner Dekankollegen ersetzen das Mitarbeiter/innengespräch durch ein Gruppengespräch mit ihren Institutsleiter/innen. Ich denke, dies ist ein Fehler, der vielerlei Möglichkeiten verschenkt. Mitarbeiter/innengespräche in einem dyadischen Setting können tiefer gehen, können die Arbeitsbeziehung fokussieren und Kritik ermöglichen, ohne vor einer universitären Quasi-Öffentlichkeit zu beschämen.

\section{Schlussfolgerung}

Dieser Erfahrungsbericht einer Dekanin und Institutsleiterin zeigt eindrucksvoll die Möglichkeiten der neuen Management-Strukturen und -Techniken, insbesondere des Mitarbeiter/innengesprächs, wenn es als Maßnahme nicht isoliert steht, sein Sinn verstanden und es aus Überzeugung von einer nicht nur auf wissenschaftlicher Ebene qualifizierten Führungskraft durchgeführt wird. Darüber hinaus wird die Bedeutung des 
Mitarbeiter/innengesprächs als unterstützendes Instrument für das Personal in einer veränderten organisationalen Realität deutlich: Teilweise übersteigerte Anforderungen einer „Weltklasse-Universität" und daraus resultierender Leistungsdruck entstehen nicht durch die Durchführung von Mitarbeiter/innengesprächen, sondern können dadurch vielmehr relativiert und auf ein vernünftiges $M a ß$ zurückgeschraubt werden. Als ein Ausgleich zum Top-Down-Management ist in kompetent gestalteten Mitarbeiter/innengesprächen ein für beide Seiten konstruktiver, wechselseitiger Austausch möglich.

Es sind also weniger Schwächen des Instruments als mangelnde Führungs- und Kommunikationskompetenz, die dazu führen, dass Mitarbeiter/innengespräche an Universitäten oft nicht den gewünschten motivierenden Effekt erzielen. Ein Umstand dem vor allem durch eine Anpassung der Auswahlverfahren für Führungskräfte sowie durch professionelle Unterstützung für Personen in Leitungspositionen, beispielsweise in Form von Coaching, begegnet werden kann.

\section{Zusammenfassung}

Der Beitrag beschreibt die verpflichtende Einführung von Mitarbeiter/innengesprächen an Österreichs Universitäten nach Einführung des neuen Universitätsgesetzes UG 2002. Wir fokussieren den kulturellen Wandel, der mit der starken Wettbewerbsorientierung der Universitäten untereinander einhergeht, und verfolgen den Prozess der Implementierung von Zielvereinbarungen und Mitarbeiter/innengesprächen auf der organisationstheoretischen, kommunikationstheoretischen und der psychodynamischen Ebene. Möglichkeiten und Grenzen der veränderten Managementpraxis an Universitäten werden diskutiert.

Schlüsselbegriffe: Universitätsmanagement, Mitarbeitergespräch, Change-Management, Coaching.

Abstract: The introduction of staff appraisal interviews in Austrian Universities

This paper addresses the issue of the obligatory introduction of staff appraisal interviews in the Austrian higher education system after the implementation the law „UG 2002“ (University Law 2002). It focuses on the cultural change process from the old university management system to a nowadays prevalent „management by objectives“. After the introduction of the „UG 2002“ Austrian Universities are forced to be more competitive with one another, hence an academic market is opened. Ultimately this leads to a cultural break. This is the reason why we discuss the possibilities and limitations of the implementation of appraisal interviews and target agreements from different angles. The diverse perspectives will be a theory of communication, a theory of organizations and a psychodynamic standpoint.

Key words: University management, staff appraisal interviews, change management, Coaching.

\section{Literatur}

Argyris, C., Schön, D.A. (1999): Die Lernende Organisation. Grundlagen, Methode, Praxis. Stuttgart: Klett-Cotta.

Brand, K.W. (1997): Probleme und Potentiale einer Neubestimmung des Projekts der Moderne unter dem Leitbild „Nachhaltige Entwicklung“. In.: Ders. (Hg.): Nachhaltige Entwicklung. Eine Herausforderung an die Soziologie. S. 9-32. Opladen: Westdeutscher Verlag.

Briggs, J., Peat, F.D. (1990): Die Entdeckung des Chaos. Eine Reise durch die Chaos-Theorie. Frankfurt/M., Wien: Dtv.

Cornforth, C. (Hg.) (2003): The Governance of Public and Non-Profit Organizations. London/ New York: Routledge.

Fritz, R. (1999). Den Weg des geringsten Widerstandes managen. Stuttgart: Klett-Cotta.

Giesecke, M. (1996): Die notwendige Integration individueller, kollektiver und institutioneller Leitbilder zu ambivalenten CI-Konzeption. Supervision 30, 72-88. 
Gröger, M. (2004): Projektmanagement. Abenteuer Wertevernichtung. Eine Wirtschaftlichkeitsstudie zum Projektmanagement in deutschen Organisationen. München: Rundfunkinterviews.

Grossmann, R., Pellert, A., Gotwald, V. (2000): Krankenhaus, Schule, Universität: Charakteristika und Optimierungspotentiale. In: Grossmann, R. (Hg.): iff Texte, Bd. 2. Besser Billiger Mehr. Zur Reform der Expertenorganisation Krankenhaus, Schule, Universität. Wien, New York: Springer, S. 24-36.

Habermas, J. (1981): Theorie des kommunikativen Handelns (Bd.1: Handlungsrationalität und gesellschaftliche Rationalisierung, Bd. 2: Zur Kritik der funktionalistischen Vernunft). Frankfurt/M.: Suhrkamp.

Hartley, J. (2002): Organizational change and development; In: Warr, P. (Ed.): Psychology at work. London, New York, p. 399-426.

Hochschild, A.R. (1990): Das gekaufte Herz. Zur Kommerzialisierung der Gefühle. Frankfurt/M.: Campus.

Höllinger, S., Titscher S. (Hg.) (2004): Die österreichische Universitätsreform. Zur Implementierung des Universitätsgesetzes 2002. Wien: WUV Universitätsverlag.

Looss, W. (1991): Coaching für Manager. Problembewältigung unter vier Augen. Landsberg am Lech: Verlag Moderne Industrie.

Luhmann, N. (1992): Universität als Milieu: Kleine Schriften. Bielefeld: Haux.

- (2002): Einführung in die Systemtheorie (hg. von Dirk Baecker). Heidelberg: Carl-AuerSysteme.

March, J.G., Olsen, J.P. (1986): Ambiguity and Choice in Organisations (2nd Ed.). Bergen: Scandinavian University Press.

Mark-Ungericht, B., Weiskopf, R. (1993): Menschenfertigung und Personalproduktion. Eine ideologiekritische kommunikationstheoretische Betrachtung von Unternehmenskultur und Personalentwicklung. Dissertation an der Leopold-Franzens-Universität Innsbruck.

Mead, G. (1994): The self. In: Clark, H., Chandler, J., Barry, J. (eds.): Organisation and Identities. London, Glasgow, New York: International Thompson, p. 99-106.

Möller, H. (2001): Teamsupervision in sterbenden Organisationen. OSC 8 (3), 283-289.

Morgan, G. (1997): Images of organization (2nd ed.). Thousand Oaks, London, New Delhi: Sage Publications.

Nonaka, I. (1994): A dynamic theory of organizational knowledge creation. Organisation Science $5(1), 14-37$.

Nonaka, I., Takeuchi, H. (1995): The Knowledge-Creating Company. Oxford: Oxford University Press.

Pellert, A. (1999): Universität als Organisation. Die Kunst, Experten zu managen. Wien, Köln, Graz: Böhlau.

Preglau, M. (1997): Kritische Theorie: Jürgen Habermas. In: Morel, J. et al. (Hg.) (1997): Soziologische Theorie. Abriss der Ansätze ihrer Hauptvertreter. München, Wien : Oldenbourg.

Rhodes, R.A.W. (Hg.) (1991): New Public Management. Public Administration, Special Issue $1 / 1991$.

Schmidt, A. (2000): Mit Haut und Haaren. Die Instrumentalisierung der Gefühle in der neuen Arbeitsorganisation. Denkanstöße, Mai 2000, 25-42.

Schön, D.E. (1983): The reflective practitioner. How professionals think in action. New York: Basic Books.

Schreyögg, A. (1996): Coaching. Eine Einführung für Praxis und Ausbildung. 2. Auflage. Frankfurt/M., New York: Campus.

Schülein, J.A. (1977): Psychotechnik als Politik. Zur Kritik der Pragmatischen Kommunikationstheorie (2. Aufl.). Frankfurt/M.

Staehle, W.H. (1999): Management. Eine verhaltenswissenschaftliche Perspektive (8. Aufl.). München: Vahlen.

Thom, N., Tholen, B. (2004): Förderung der Management-Kompetenz für Dozierende und Führungskräfte der Universität Bern. In: Laske, S., Scheytt, T., Meister-Scheytt, C. (Hg.): Perso- 
nalentwicklung und universitärer Wandel: Programm - Aufgaben - Gestaltung, S. 355-378. München, Mering: Rainer Hampp.

Volkan, V.D., Ast, G. (1994): Spektrum des Narzissmus. Göttingen: Vandenhoeck \& Ruprecht. Watzlawick, P., Weakland, J.H., Fisch, R. (1992): Lösungen. Zur Theorie und Praxis menschlichen Wandels (5. Aufl.). Bern u.a.: Hans Huber.

Weick, K.E., Quinn, R.E. (1999): Organizational change and development. Annual Review of Psychology, 50, p. 361-386.

Winnicott, D.W. (1991): Von der Kinderheilkunde zur Psychoanalyse. Frankfurt/M.: Fischer.

Die Autor/innen:

Gabriela Edlinger, Mag., Akademische Kommunikationsberaterin, Sozial- und Wirtschaftswissenschafterin, wissenschaftliche Mitarbeiterin am Institut für Organisation und Lernen der Fakultät für Betriebswirtschaft, Universität Innsbruck. Anschrift: Institut für Organisation und Lernen, Universitätsstraße 15, A-6020 Innsbruck, E-Mail: Gabriela.Edlinger@uibk.ac.at.

Claudia Meister-Scheytt, Dipl.-Ök., Industriekauffrau, Wirtschaftswissenschaftlerin, Organisationsberaterin, wissenschaftliche Mitarbeiterin am Institut für Organisation und Lernen der Fakultät für Betriebswirtschaft, Universität Innsbruck. Anschrift: Institut für Organisation und Lernen, Universitätsstraße 15, A-6020 Innsbruck, E-Mail: Claudia.Meister-Scheytt@uibk.ac.at.

Heidi Möller, Prof. Dr., Dipl.-Psych., Psychoanalytikerin, Organisationsberaterin, Supervisorin, Lehrtherapeutin für Tiefenpsychologie und Gestalttherapie Lehrsupervisorin, Leiterin des Instituts für Professorin für Kommunikation im Berufsleben und Psychotherapie, Dekanin der Fakultät für Bildungswissenschaften, Universität Innsbruck. Anschrift: Institut für Kommunikation im Berufsleben und Psychotherapie, Schöpfstraße 3, A-6020 Innsbruck, E-Mail: Heidi.Moeller@ uibk.ac.at 